\title{
Síndrome confusional: échale la culpa a los opioides...
}

\author{
M. Fernández Hernández¹, R.M. Santillán Fernández¹, E. Rodríguez Rodríguez², D. Bouzas Pérez ${ }^{3}$ \\ y J.M. Carceller Malo
}

\author{
${ }^{I}$ Servicio de Anestesiología, Reanimación y Terapéutica del Dolor. ${ }^{2}$ Servicio de Neurología. Hospital \\ Universitario Marqués de Valdecilla. Santander. ${ }^{3}$ Medicina Física y Rehabilitación. Hospital de Laredo. \\ Cantabria
}

Fernández Hernández M, Santillán Fernández RM, Rodríguez Rodríguez E, Bouzas Perez D, Carceller Malo JM. Síndrome confusional: échale la culpa a los opioides.... Rev Soc Esp Dolor 2015; 22(2): 62-68.

\begin{abstract}
A 42-year-old male with precedents of alcoholism, abuse of cocaine and fracture of right tibial plateau of six months of evolution that was treated surgically, at a first time, and required multiple reinterventions for infection of the surgical wound and osteomielitis. The patient presented depressive sindrome and was polymedicated with endovenous antibiotics and opioids, and oral formulations of benzodiazepines, analgesics and rescue medicaments (distraneurine, bromazepam, pregabalina, paracetamol, metamizol, tramadol, Enantyun ${ }^{\circledR}$ and morphine). The patient was presenting especially pain of neuropathic type with a VAS of 8 , so benzodiazepines were suspended and analgesia is changed by oral route: Pregabalina (150 mg-0-150 mg), amitriptilina (0-0-25 mg), Oxycontin ${ }^{\circledR}(10-0-10$ $\mathrm{mg}$ ) and paracetamol $1 \mathrm{~g} / 8 \mathrm{~h}$. When the decrease of opioids dose was possible for good pain control,the patient presents an important stupor in the night, without answering to stimuli and obey orders, which it alternates with important psychomotor agitation, incoherence in language and disconnection with the environment. This syntomatology persists.

The habitual doctors of the patient were believing in an overdosage of opioids as origin of the syntomatology.

In this case we would have to make a differential diagnosis with the principal reasons of confusional syndrome with: Blood poisoning, endocarditis, cerebral abscesses or stroke, orthopae-
\end{abstract}

Recibido: 15-03-14.

Aceptado: 22-06-14 dic (hip and knee), postoperatory delirium, drugs (opioids or triciclics).

The polymedication was suspected as main cause of the syntomatology, though the only thing that seems to be clear is that the psychomotor agitation was due to an abstinence syndrome to opioids, but it was necessary to look for another reason for the repeated decrease of the level of conscience. The postoperatory delirium is very feasible in this patient, though the consulted literature does not give great evidence on which medication used for the anesthesias could caused the confusional syndrome.

A great number of alterations of the behavior are associated with cerebral strokes in the territory of the cerebral artery right average. A confucional syndrome in 13-48\% of the patients in the acute phase of a cerebral stroke. A $12 \%$ of the cerebral strokes occurs in patients younger of 45-year-old, being the way of presentation of very diverse systemic or local diseases, nonetheless, in $40 \%$ of the cases are unnoticed.

The confusional syndrome is underdiagnosed. The false myths on the opioids, the opiofobia and the low knowledge of this medication carry to a therapeutic breach by the patients and/or sanitary personnel. The syndrome of abstinence has to be counted when we diminish dose of opiods taken chronically. It is important to follow a scheme of differential diagnosis to assure ourselves the accurate diagnosis of the underlying patho$\log y$.

Key words: Confusional syndrome. Opioids. Cerebral stroke.

\section{RESUMEN}

Varón de 42 años con antecedentes de alcoholismo, abuso de cocaína y fractura de meseta tibial derecha de seis meses de evolución que se trato quirúrgicamente, inicialmente, y precisa de múltiples reintervenciones por infección de la herida quirúrgica y osteomielitis.

El paciente presentaba ánimo depresivo y estaba polimedicado: antibióticos endovenosos y opioides, BZD, analgésicos y fármacos coadyuvantes v.o. (distraneurine, bromazepam, pregabalina, paracetamol, metamizol, tramadol, Enantyun ${ }^{\circledR}$ y morfina). 
El paciente presentaba sobre todo dolor de tipo neuropático con un EVA de 8, por lo que se suspenden las BZD y se pauta analgesia por vía oral: pregabalina (150 mg-0-150 mg), amitriptilina (0-0-25 mg), Oxycontin ${ }^{\circledR}$ (10-0-10 mg) y paracetamol $1 \mathrm{~g} / 8 \mathrm{~h}$. Cuando se plantea la disminución de la dosis de opioide por buen control del dolor, debuta con un cuadro de estupor importante por la noche, sin responder a estímulos ni obedecer órdenes, que alterna con agitación psicomotriz importante, incoherencia en el lenguaje y desconexión con el medio. Este cuadro persiste.

Los médicos habituales del paciente creían en una sobredosificación de opioide como origen del cuadro.

En este caso tendríamos que hacer un diagnóstico diferencial con las principales causas de síndrome confusional con: septicemia, endocarditis, abscesos cerebrales, ortopédica (cadera y rodilla), delirio postoperatorio, toma de opioides o tricíclicos.

La polimedicación era sospechada como causa principal del cuadro, si bien lo único que parece claro es que la agitación psicomotriz se debía a un síndrome de abstinencia a opioides, pero había que buscar otra causa para la disminución reiterada del nivel de consciencia.

El delirio postoperatorio es muy factible en este paciente, aunque la literatura consultada no aporta mucha evidencia sobre que la medicación usada para las anestesias de este paciente sean las precipitantes de este cuadro.

Una gran variedad de alteraciones del comportamiento se ven asociadas a infartos cerebrales en el territorio de la arteria cerebral media derecha. El síndrome confusional aparece en el 13-48 \% de los pacientes en la fase aguda de un infarto cerebral. Un $12 \%$ de los ACVA se dan en pacientes menores de 45 años, siendo el modo de presentación de muy diversas enfermedades sistémicas o locales, aún así, en el $40 \%$ de los casos no se puede diagnosticar la etiología.

El deliro es un cuadro infradiagnosticado. Los falsos mitos sobre los opioides, la opiofobia y el escaso conocimiento de esta medicación conllevan a un incumplimiento terapéutico por parte de los pacientes y/o del personal sanitario. El síndrome de abstinencia ha de tenerse siempre en cuenta cuando disminuimos la dosis de opioide que se toma de forma crónica.

Es importante seguir un esquema de diagnóstico diferencial, ya que con ello nos aseguramos el diagnóstico certero de la patología subyacente.

Palabras clave: Síndrome confusional. Opioides. ACVA.

\section{CASO CLÍNICO}

Varón de 42 años con antecedentes personales de alcoholismo, abuso de cocaína y fractura de meseta tibial derecha en enero de 2011 que se trató quirúrgicamente (osteosíntesis-placa con injerto cresta iliaca). Se produce una infección de herida quirúrgica que acaba generando una osteomielitis tibial derecha a los 4 meses, requiriendo varias intervenciones quirúrgicas para extracción de material de osteosíntesis, desbridamiento y limpieza.

El paciente está ingresado desde el $4 .^{\circ}$ mes y en tratamiento con antibioterapia endovenosa de última generación que se administra por una vía central, y vía oral con 2 comprimidos de distraneurine y bromazepam $/ 12 \mathrm{~h}$ desde su ingreso (para prevenir un delirium por deprivación alcohólica); como analgesia: pregabalina 75-0-150, paracetamol $1 \mathrm{~g} / 8 \mathrm{~h}$ endovenosa, metamizol $2 \mathrm{~g} / 8 \mathrm{~h}$ endovenosa, tramadol $100 \mathrm{mg} / 8 \mathrm{~h}$ endovenosa, Enantyun ${ }^{\circledR} 25 / 8$ h vía oral y morfina $5 \mathrm{mg} / 6 \mathrm{~h}$ endovenosa (un total de $20 \mathrm{mg}$ endovenosos al día).

$\mathrm{Al}$ 8..$^{\circ}$ mes de evolución del proceso interconsultan a la Unidad del Dolor por mal control del dolor (Tabla I).

$\mathrm{Al}$ valorar al paciente se encontraba con ánimo depresivo y con un dolor insoportable, con un 8 en la escala visual analógica (EVA 8), de tipo neuropático -quemazón y pinchazos intensos en extremidad inferior derecha (EID)que le dificultaba el descanso nocturno e incluso le despertaba. En este momento también presentaba picos febriles de hasta $39^{\circ} \mathrm{C}$ registrados en la gráfica de enfermería, sin repercusión orgánica.

El manejo del tratamiento del dolor de este paciente resultó sencillo en un principio, pues presentaba sobre todo dolor de tipo neuropático. Por nuestra parte planificamos suspender escalonadamente el distraneurine y el bromazepan. Se pasó de la analgesia endovenosa a la vía oral, conseguiéndose una disminución de la sintomatología: añadiendo y aumentando antineuropáticos $(1,2)$ : pregabalina (hasta llegar a $150 \mathrm{mg}-0-150 \mathrm{mg}$ ) asociada con amitriptilina (0-0-25 mg). Suspendiendo la morfina endovenosa y cambiando a Oxycontin ${ }^{\circledR}$ a dosis equianalgésicas con la morfina (10-0-10 mg) y paracetamol $1 \mathrm{~g} / 8 \mathrm{~h}$, precisando puntualmente rescates con Oxynorm ${ }^{\circledR} 5 \mathrm{mg}$.

Al cuarto día del seguimiento el paciente debutó con un cuadro de estupor importante por la noche y al día siguiente, sin responder a estímulos ni obedecer órdenes. Tras descartar consumo exógeno de opioide, se disminuye la dosis total de opioide a MST ${ }^{\circledast} 10-0-10$ y Adolonta ${ }^{\circledR} 100$ mg de rescate si dolor (precisando al inicio del tratamiento una antes de acostarse y ocasionalmente a lo largo del día, y no precisándola al cabo de 10 días).

Al quinto día el paciente se encuentra con un cuadro de agitación psicomotriz importante, incoherencia en el lenguaje y desconexión con el medio, compatible con el diagnóstico de síndrome de abstinencia.

Nos vemos obligados a realizar un diagnóstico difencial (Fig. 1). Descartado el origen metabólico del cuadro confusional (analítica normal), se piensa en un síndrome de sobredosis/abstinencia, para algunos originado por la dosis de opioide pautado y para nosotros por un posible abuso de sustancias (el perfil de tóxicos en orina no es concluyente a este respecto, pues presentaba positividad para las sustancias ya pautadas y negatividad para el cannabis), por lo que se realiza una interconsulta a psiquiatría para que valore al paciente y confirme si hay un patrón de abuso de sustancias (la familia niega consumo de tóxicos por parte del paciente, pero no siempre están con él) u otro origen. 
TABLA I. CRONOLOGÍA DE LOS EVENTOS

\begin{tabular}{|c|c|c|c|}
\hline & Fecha & Evento & Tratamiento \\
\hline 1 & 12 de enero 2011 & Fractura meseta tibial & Osteosíntesis-placa con injerto de cresta iliaca \\
\hline 2 & 10 de marzo de 2011 & Infección de herida quirúrgica & EMO tibia dcha. + desbridamiento, en H. Sierrallana \\
\hline 3 & 13 de abril 2011 & Mala evolución herida quirúrgica & $\begin{array}{l}\text { Traslado desde H. Sierrallana para ingreso en } \\
\text { plástica en HUMV }\end{array}$ \\
\hline 4 & 28 de abril de 2011 & Osteomielitis meseta tibial & Cambio cabecera a Traumatología \\
\hline 5 & 3 de junio de 2011 & Intervención quirúrgica & $\begin{array}{l}\text { Secuestrectomía + desbridamiento + espaciador de } \\
\text { cemento con antibiótico + toma de cultivos }\end{array}$ \\
\hline 6 & 23 de junio de 2011 & Intervención quirúrgica & Trasposición de gemelo interno \\
\hline 7 & 7 de julio de 2011 & Intervención quirúrgica con $\mathrm{AG}^{*}$ & $\begin{array}{l}\text { Desbridamiento + recambio de espaciadores }+ \text { toma } \\
\text { de cultivos }\end{array}$ \\
\hline 8 & 4 de agosto de 2011 & Dolor mal controlado & Unidad del Dolor ajusta tratamiento \\
\hline 9 & 5 de agosto de 2011 & Intervención quirúrgica con AG & $\begin{array}{l}\text { Desbridamiento + espaciador con cemento y } 2 \text { barras } \\
\text { de FE + toma de cultivos }\end{array}$ \\
\hline 10 & 8 de agosto de 2011 & Sd. confusional & Ajuste de medicación \\
\hline 11 & 14 de agosto de 2011 & Intervención quirúrgica con $\mathrm{AG}$ & $\begin{array}{l}\text { Retirada espaciador cemento y barras }+ \text { FE } \\
\text { Hoffman II puenteando la rodilla }\end{array}$ \\
\hline 12 & 21 de agosto de 2011 & Intervención quirúrgica con $\mathrm{AG}$ & $\begin{array}{l}\text { EMO FE + EMO clavo gamma corto + clavo gamma } \\
\text { largo + clavo GK + espaciador de cemento }\end{array}$ \\
\hline 13 & 25 de agosto de 2011 & Persistencia sd. confusional & TAC craneal \\
\hline
\end{tabular}

*AG: anestesia general.

La impresión diagnóstica del psiquiatra fue polimedicación, sobre todo los opioides, y sugieren una benzodiacepina de vida media corta para controlar la agitación psicomotriz (alprazolam $4 \mathrm{mg}$ v.o./8 h, que se fue disminuyendo progresivamente según iba remitiendo el cuadro). A este respecto, como ya se había disminuido el opioide, no se tomó ninguna otra medida.

En cuanto al control del dolor, en los siguientes 15 días, el tratamiento antineuropático fue muy efectivo, excepto por una persistencia de dolor en forma de "pinchazos", que requirió de un aumento gradual de la amitriptilina $(1,2)$ hasta $75 \mathrm{mg}$ por la noche. Esta medida consiguió un EVA2, con una importante mejora en el descanso nocturno y en el estado de ánimo. Además, el paciente deja de precisar rescates, como ya se ha comentado.

Por ello nos podríamos haber planteado la disminución de la medicación opioide, pero el paciente seguía presentando alteraciones del comportamiento y del nivel de consciencia que iban del estupor a la agitación psicomotriz, teniendo como primera repercusión la no administración del opioide pautado por parte del personal de enfermería, por miedo a que fuese una sobredosis de opioide y que esta conllevase una depresión respiratoria, y en segundo lugar requiriendo contención mecánica tras caerse y luxarse el espaciador colocado en fémur.
Se insiste en que se ha de administrar toda la medicación pautada y se mantiene la dosis total de opioide (MST ${ }^{\circledR}$ 10-0-10 y Adolonta ${ }^{\circledR} 100 \mathrm{mg}$ de rescate), para no reincidir en posibles síndromes de abstinencia.

A pesar de que el paciente presentaba picos febriles, los infectólogos que seguían al paciente no consideraban que la fiebre ni otra posible complicación derivada de la osteomielitis, como una endocarditis o absceso cerebral, fuesen responsables del cuadro.

Como nos parecía poco probable que una sobredosificación/abstinencia de los opioides pautados fuese el origen del síndrome confusional que padecía este paciente a lo largo de más de 2 semanas, se decide descartar organicidad pidiendo un TAC craneal a las 3 semanas del seguimiento.

En el TAC craneal se visualizó un infarto cerebral parietal derecho subagudo (Fig. 2).

\section{DISCUSIÓN}

El síndrome confusional o delirio (Tabla II) se caracteriza por un cuadro de instauración aguda de alteración del nivel de consciencia, memoria, pensamiento y comportamiento. Hay muchos factores predisponentes para que se 


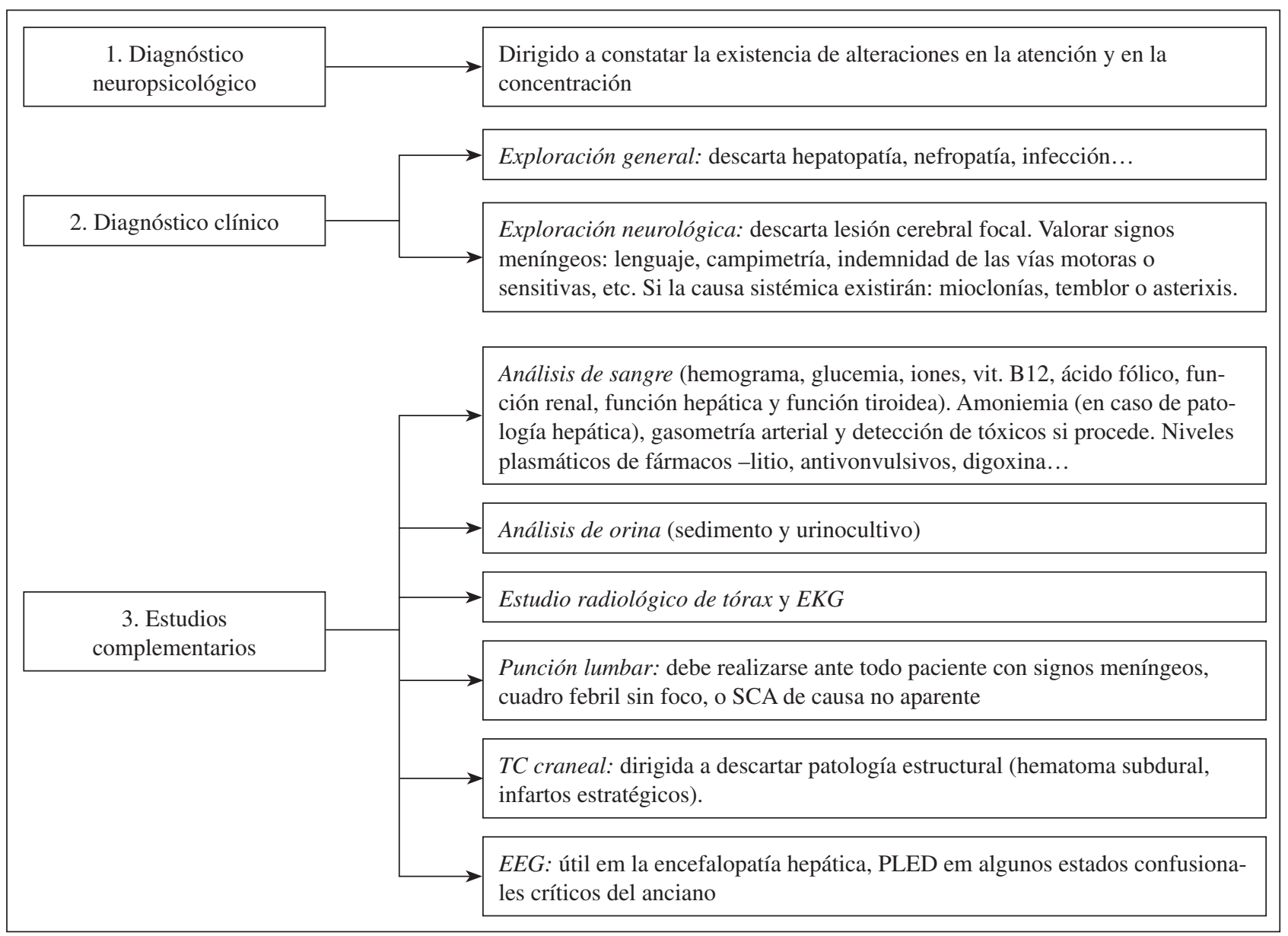

Fig. 1. Diagnóstico diferencial del síndrome confusional agudo.
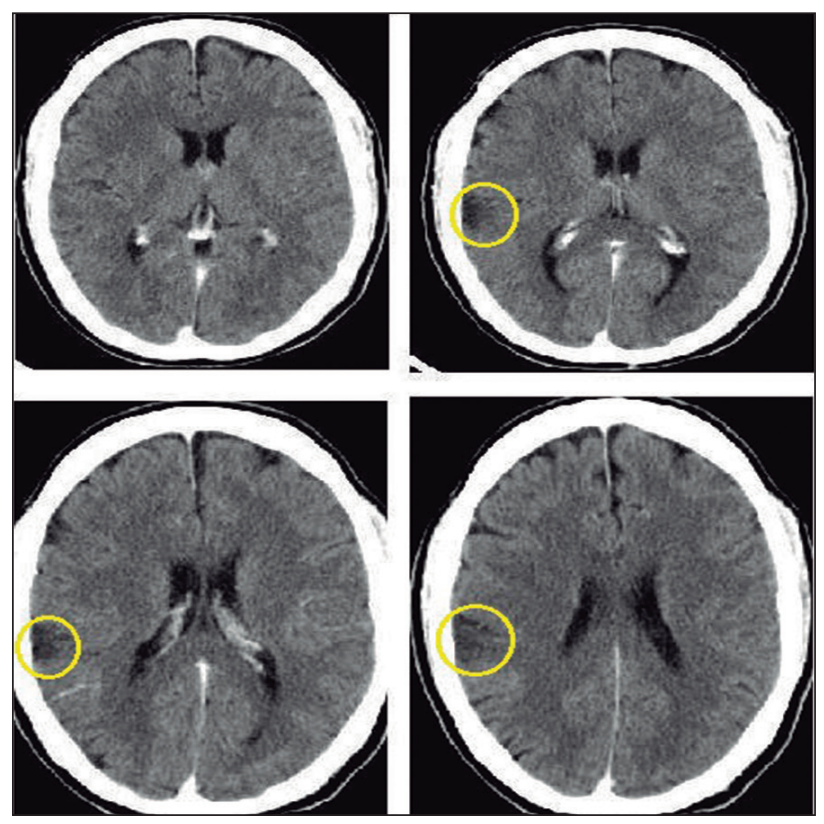

Fig. 2. TAC craneal. dé este cuadro: edad avanzada, infección urinaria silente o alteraciones cognitivas previas. Las principales causas se muestran en la tabla III.

Para descartar una sobredosis por abuso de sustancias como origen de la disminución del nivel de consciencia y por otro lado un síndrome de abstinencia como causa de la agitación psicomotriz, se solicitaron tóxicos en orina que dieron positivos para opioides, antidepresivos y benzodiacepinas, siendo negativo para anfetaminas, cocaína, cannabinoides, barbitúricos y metadona (el test no diferencia entre morfina y heroína). Aunque no se pudo confirmar este origen como causa de los síntomas tampoco pudimos descartarlo del todo.

La polimedicación era sospechada como causa principal por todos los médicos que veían al paciente: traumatólogos, infectólogos y psiquiatras. En el momento de aparecer el cuadro el paciente tomaba: Oxycontin ${ }^{\circledast} 10-0-10$, amitriptilina 25 en la cena, tramadol $50 \mathrm{mg} / 8 \mathrm{~h}$ si dolor, paracetamol 1 g/8 h v.o. y Enantyum ${ }^{\circledR} 25$ mg v.o./8 h. Desde nuestro punto de vista esta medicación era poco probable que causara una disminución tán brusca del nivel del consciencia sin otra repercusión (depresión respiratoria) y 
TABLA II. CRITERIOS DIAGNÓSTICOS DEL SÍNDROME CONFUSIONAL (DSM IV) (3)

- Alteración de la consciencia (menor alerta al entorno) con disminución de la capacidad para centrar, mantener o dirigir la atención

- Cambio en las funciones cognitivas (déficit de memoria, desorientación, alteración del lenguaje) o aparición de una alteración de la percepción no explicable por la existencia de una demencia preexistente o en desarrollo

- Su aparición se presenta en un corto periodo de tiempo (horas o días) y tiende a fluctuar durante el día

- Demostración, a través de la historia clínica, de la exploración física o de las pruebas de laboratorio, que el delirio se debe a efectos fisiológicos directos de una enfermedad médica, de la intoxicación o la privación de sustancias, del consumo de medicamentos o de la exposición a tóxicos, o bien a una combinación de estos factores

menos aún que la alteración del nivel de consciencia persistiese durante 20 días.

Lo que visto ahora parece claro es que la agitación psicomotriz se debía a un síndrome de abstinencia a opioides: en un primer momento por la disminución de la dosis de opioide a la mitad y en un segundo lugar por la no administración de dos dosis del opioide pautado. Pero en aquel momento no nos pareció que el conjunto del cuadro de disminución del nivel de consciencia-agitación psiomotriz nos obligaba a descartar la existencia de otra causa, por lo que seguimos con el diagnóstico diferencial.

El delirio postoperatorio (6) es muy factible en este paciente. Como factores de riesgo para este cuadro se han publicado diferentes estudios que definen que la presencia de más de dos de estos factores aumenta el riesgo (edad avanzada, bajo minimental, episodios previos, consumo de alcohol o benzodiacepinas, incapacidad para alguna de las actividades de la vida diaria, estancia larga en hospitales, procesos concomitantes) de presentar un delirio postoperatorio. Este cuadro no se diagnostica en un $66 \%$ de los casos. Puede aparecer desde el despertar (se resuelve en minutos u horas), o posteriormente con intervalo lúcido de 24-72 h previo, tener un curso fluctuante a lo largo del día, siendo la duración y la severidad de este cuadro variable. Este paciente sufrió una intervención en las 72 horas previas a la aparición del síndrome confusional. Aunque él presentaba dos factores de riesgo asociados para este cuadro (toma crónica de bromazepam y estancia larga en hospital) y para la intervención quirúrgica se realizaba una anestesia general en la que se usó midazolan, propofol, desfluorane y Fentanest ${ }^{\circledR}$, la literatura consultada $(7,8)$ ofrece ciertos matices que debilitan esta teoría como origen del cuadro. Así bien, las benzodiacepinas

TABLA III. PRINCIPALES CAUSAS DEL SÍNDROME CONFUSIONAL AGUDO (4)

\begin{tabular}{ll}
\hline & Hipoxia, hipoglucemia, hipertiroidismo, mixedema, crisis addisonianas, insuficiencia \\
& renal, hepática y pancreática \\
Metabólicas & Deshidratación, hipo o hipercalcemias, hipo o hipermagnesemia, hipo o hipernatremia, \\
& acidosis, alcalosis e hipercapnia \\
- & Déficit de tiamina y cianocobalamina \\
- & Alcohol, monóxido de carbono \\
- & No neurológicas: infección de orina, neumonía, bacteriemia, septicemia, endocarditis \\
- & Neurológicas: meningitis, encefalitis, abscesos cerebrales \\
- & Infarto agudo de miocardio, tromboembolismo pulmonar \\
Infecciones & Infarto cerebral (sobre todo en lóbulo parietal y región inferior medial del lóbulo \\
& occipital), hemorragia subaracnoidea, enecefalopatía hipertensiva, migraña, vasculitis \\
Alteraciones vasculares & - Cardiaca, ortopédica (cadera y rodilla), catarata, resección transuretral/prostática. Delirio \\
& postoperatorio (6) \\
Cirugías & Opioides: codeína, meperidina \\
- & Hipnóticos sedantes: fenobarbital, alprazolam, diazepam... \\
- & Neurolépticos: fenotiacinas, clozapina \\
- & Anticolinérgicos: antihistamínicoas, tricíclicos, antiespasmódicos \\
Fármacos & Síndrome de hiperviscosidad, discrasias sanguíneas, golpes de calor, electrocución, \\
& traumatismo caneoencefálico, encefalopatía de Wernicke, amnesia global transitoria, \\
& epilepsia o periodo postcrítico... \\
\hline
\end{tabular}




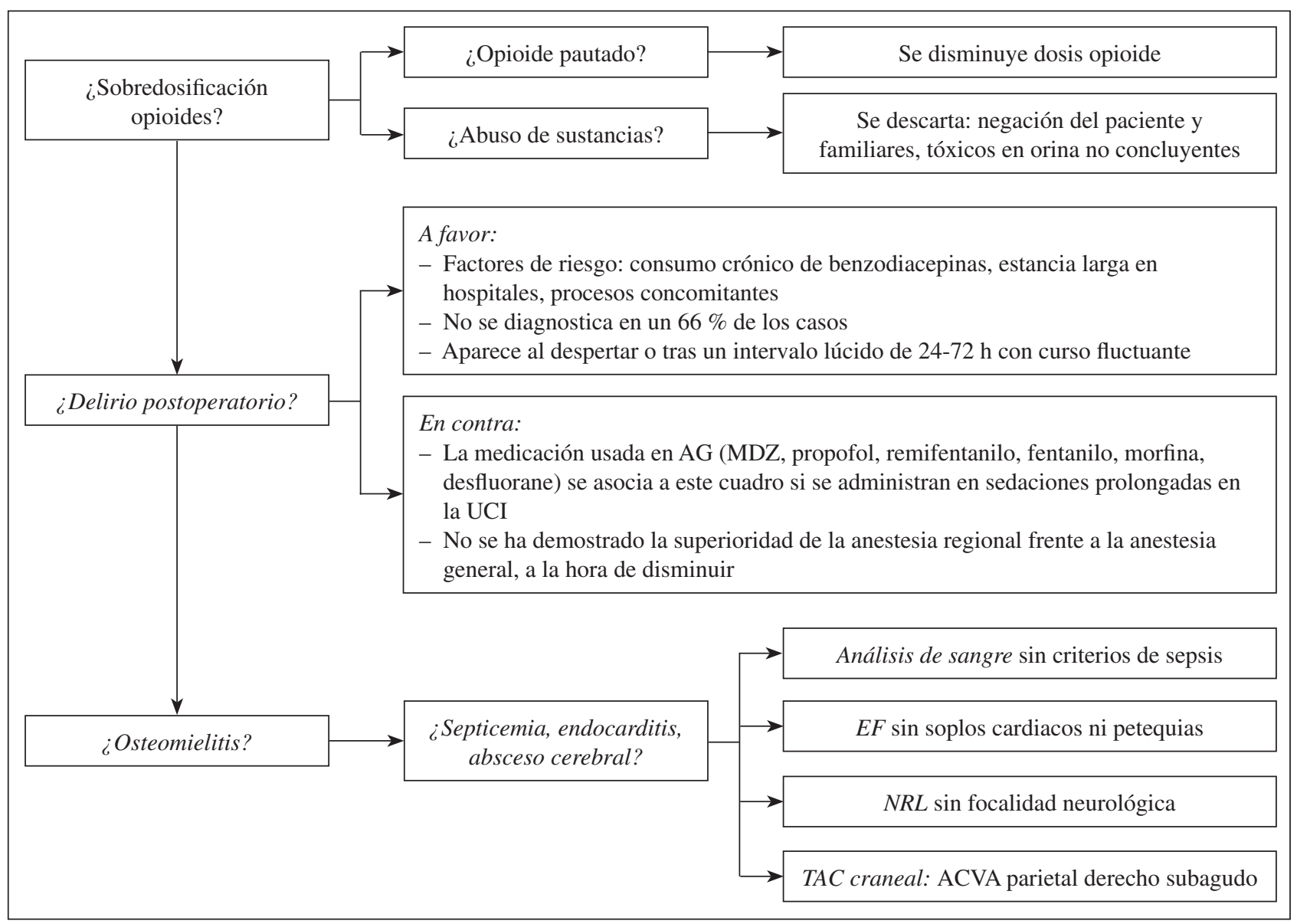

Fig. 3. Posibles etiologías y proceso diagnóstico del síndrome confusional en nuestro caso.

son desencadenantes de deliro si se da una administración crónica de las mismas y más si son de vida media larga, el propofol usado como mantenimiento de la anestesia frente al desfluorane presenta mayor frecuencia de delirio, el fentanilo y la morfina (a dosis mayores de $10 \mathrm{mg}$ ) tienen mayor grado de asociación a delirio que el remifentanilo. Por otro lado, no se ha desmotrado los superioridad de la anestesia regional frente a la anestesia general, a la hora de disminuir la aparición del delirio postoperatorio.

El hallazgo de un accidente cerebrovascular isquémico en el TAC fue, desde luego, inesperado, pero se ha de tener en cuenta que una gran variedad de alteraciones del comportamiento se ven asociadas a infartos cerebrales en el territorio de la arteria cerebral media derecha (9), entre ellos el síndrome confusional. El síndrome confusional $(8,9)$ aparece en el 13-48 \% de los pacientes en la fase aguda de un infarto cerebral, comparado con el 10-25\% que aparece en los pacientes ingresados en medicina. Estos pacientes presentan una probabilidad aumentada de 4 , 7 veces de morir durante el ingreso o en los 12 meses posteriores al evento. Además, estos pacientes tienen mayor índice de dependencia al alta.
Un $12 \%$ de los ACVA se dan en pacientes menores de 45 años $(12,13)$, en este grupo de población pueden ser el modo de presentación de muy diversas enfermedades sistémicas o locales: estenosis carotídea, ateromatosis, endocarditis... aún así, en el $40 \%$ de los casos no se puede diagnosticar la etiología. Los factores de riesgo (12) más comunes de isquemia cerebral (HTA, DM) no suelen estar presentes en los adultos jóvenes, sin embargo sí suelen presentar otros factores de riesgo: tabaquismo, anticonceptivos orales, migrañas, traumas, abuso de sustancias (13\%), consumo crónico de alcohol o cocaína, puerperio o embarazo. El pronóstico, y la mortalidad, están en relación con la gravedad de las patologías subyacentes y el tratamiento oportuno de las mimas.

\section{CONCLUSIONES}

El deliro es un cuadro infradiagnosticado (14); 1 de cada 3 episodios se podría prevenir y su prevención en los grupos de riesgo disminuiría mucho los costes (menor morbilidad, menor estancia hospitalaria). 
Por un lado, los falsos mitos sobre los opioides y la opiofobia, con miedo a sus efectos secundarios, sobre todo la sobredosificación o el riesgo a poder desencadenar un síndrome de dependencia en el paciente, conllevan a un incumplimiento terapéutico por parte de los pacientes y/o del personal sanitario.

Así mismo es preocupante el escaso conocimiento del manejo de la medicación analgésica, y de los opioides más concretamente, que existe en general en el medio sanitario.

El síndrome de abstinencia ha de tenerse siempre en cuenta cuando disminuimos dosis de opioide que se toma de forma crónica.

El síndrome de abstinencia origina un gran sufrimiento al paciente y a los familiares, es por ello que el miedo a la depresión respiratoria, por sobredosificación opioidea, no puede llevarnos a no administrar la medicación, debiéndose por ello hacer más esfuerzo en la formación de los profesionales sanitarios a este respecto.

Es importante seguir un esquema de diagnóstico diferencial (Fig. 3) y no dejarse influenciar por opiniones no basadas en la evidencia científica o prejucios. El realizar un diagnóstico diferencial implica un esfuerzo enorme ya que requiere de: una anamnesis, exploración física, realización de pruebas diagnósticas e interacción entre distintos especialistas médicos, más allá de lo que se había planeado y que incrementa el consumo de los recursos disponibles. Sin embargo, con ello nos aseguramos el diagnóstico certero de la patología subyacente, pudiendo así tomarse las medidas oportunas para el bienestar del paciente.

\section{CORRESPONDENCIA:}

Marta Fernández Hernández

Servicio de Anestesiología, Reanimación y Terapeútica del Dolor

Hospital Universitario Marqués de Valdecilla

Avda. Valdecilla, s/n

39008 Santander

e-mail: martafhdez@ hotmail.com

\section{BIBLIOGRAFÍA}

1. O'Connor AB, Dworkin. Treatment of neuropathic pain: An overview of recent guidelines. Am J Med 2009 122(10 Sup:S22-32.

2. Freynhagen R, Bennett MI. Diagnosis and management of neuropathic pain. BMJ 2009;339:b3002. doi: 10.1136/bmj. b3002.

3. Manual diagnóstico y estadístico de los trastornos mentales. Barcelona: Masson SA; 1998.

4. Ruiz MI, Mateos V, Suárez H, Villaverde P. Síndrome confusional agudo (Delirium) "Guía práctica de diagnóstico y tratamiento". Hospital Universitario Central de Asturias Oviedo Principado de Asturias.

5. San Roman MD, Roca J. Síndrome confusional agudo. JANO 2008;1684:31-4.

6. Steiner LA. Postoperative delirium. Part 2: Detection, prevention and treatment. Eur J Anesthesiology 2011;28(10): 723-30.

7. Guenther U, Radtke FM. Delirium in the postanestesia period. Curr Opin Anesthesiol 2011;24:670-5.

8. Steiner LA. Postoperative delirium. Part 1: Pathophysiology and risk factors. Eur J Anesthesiology 2011;28(9):628-36.

9. Mohr JP, Choi DW, Grotta JC, Weir B, Wolf PA. Middle cerebral artery disease en STROKE: Pathophysiology, diagnosis, and manegement. $4 .^{\circ}$ ed. EEUU: Churchill Livingstone; p. 154-9.

10. Shi Q, Presutti P, Selchen D, Saposnik G. Delirium in acute stroke. A Systematic review and meta-analysis. Stroke 2012;645-9.

11. Oldenbeuving AW, de Kort PL, Jansen BP, Roks G, Kappelle LJ. Delirium in acute stroke: A review. Int J Stroke 2007;2:270-5.

12. Ferro J M, Massaro A R, Mas J-L. Aetiological diagnosis of ischaemic stroke in young adults. Lancet Neurol 2010; 9:1085-96.

13. Varona JF. Diagnostic work-up and etiology in ischemic stroke in young adults: Before and now. J Neurol Neurophysiol 2012;3(4):133-6.

14. O'Mahony R, Murthy L, Akunne A, Young J. Synopsis of the National Institute for Health and Clinical Excellence Guideline for prevention of delirium. Ann Intern Medicine 2011;154(11):746-51. 